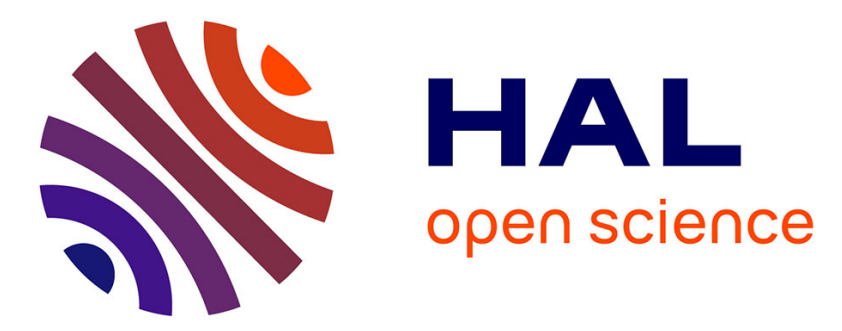

\title{
Modeling of X-ray emissions produced by stepping lightning leaders
}

Wei Xu, Sébastien Celestin, Victor P. Pasko

\section{To cite this version:}

Wei Xu, Sébastien Celestin, Victor P. Pasko. Modeling of X-ray emissions produced by stepping lightning leaders. Geophysical Research Letters, 2014, 41, pp.7406-7412. 10.1002/2014GL061163 . insu-01174373

\section{HAL Id: insu-01174373 https://hal-insu.archives-ouvertes.fr/insu-01174373}

Submitted on 9 Jul 2015

HAL is a multi-disciplinary open access archive for the deposit and dissemination of scientific research documents, whether they are published or not. The documents may come from teaching and research institutions in France or abroad, or from public or private research centers.
L'archive ouverte pluridisciplinaire HAL, est destinée au dépôt et à la diffusion de documents scientifiques de niveau recherche, publiés ou non, émanant des établissements d'enseignement et de recherche français ou étrangers, des laboratoires publics ou privés. 


\section{Geophysical Research Letters}

\author{
RESEARCH LETTER \\ 10.1002/2014GL061163 \\ Key Points: \\ - Predictions on energy spectra \\ of X-rays produced by stepping \\ lightning leaders \\ - Radial and electric potential depen- \\ dence of X-ray spectra measured at \\ the ground \\ - Using X-ray bursts to evaluate \\ the electrical properties of \\ lightning leaders
}

\section{Correspondence to:}

W. Xu,

wxx5015@psu.edu

\section{Citation:}

Xu, W., S. Celestin, and V. P. Pasko (2014), Modeling of X-ray emissions produced by stepping lightning leaders, Geophys. Res. Lett., 41, 7406-7412, doi:10.1002/2014GL061163.

Received 8 JUL 2014 Accepted 24 SEP 2014 Accepted article online 26 SEP 2014 Published online 21 OCT 2014

\section{Modeling of X-ray emissions produced by stepping lightning leaders}

\author{
Wei Xu' ${ }^{1}$, Sebastien Celestin ${ }^{2}$, and Victor P. Pasko ${ }^{1}$ \\ ${ }^{1}$ Communications and Space Sciences Laboratory, Department of Electrical Engineering, Pennsylvania State University, \\ University Park, Pennsylvania, USA, ${ }^{2}$ Laboratory of Physics and Chemistry of the Environment and Space (LPC2E), \\ University of Orleans, CNRS, Orleans, France
}

\begin{abstract}
Intense and brief bursts of X-ray emissions have been measured during the stepping process of both natural cloud-to-ground (CG) and rocket-triggered lightning flashes. In this paper, we investigate theoretically the energy spectra of X-rays produced by the bremsstrahlung emission of thermal runaway electrons accelerated in the inhomogeneous electric field produced around lightning leader tips. The X-ray energy spectrum depends on the physical properties of the associated lightning leaders. Consequently, X-ray measurements can be used for diagnostics of the electrical properties of lightning stepped leaders. We report simulation results of the photon energy spectra produced by 5 and 10 MV negative CG lightning discharges that would be measured from the ground using ideal detectors. We also quantify theoretically the radial dependence of $\mathrm{X}$-ray energy spectra received at ground level during the leader stepping process. Moreover, it is found that the ground radiation generated in this process is harmless to humans.
\end{abstract}

\section{Introduction}

Intense and very brief bursts of X-ray emissions have been detected from the ground during natural cloud-to-ground (CG) lightning flashes [Moore et al., 2001] and rocket-triggered lightning flashes [Dwyer et al., 2003]. These bursts are mainly composed of X-rays with energies extending up to a few hundreds of keV [e.g., Dwyer et al., 2004]. Measurements at the International Center for Lightning Research and Testing (ICLRT) have revealed that discrete and intense bursts of X-rays were time correlated with the formation of leader steps during CGs [Dwyer et al., 2005]. This finding was extended by Howard et al. [2008], who have spatially and temporally colocated X-ray emissions with leader step electric field changes for both downward negative natural and rocket-triggered lightning flashes.

More recently, ground-based observations have provided valuable insights to the properties of these X-rays. By imaging X-ray emissions from triggered lightning dart leaders using a pinhole-type high-speed X-ray camera (XCAM), Dwyer et al. [2011] have demonstrated that the leader front is the source of the X-ray emissions. Moreover, from the analysis of X-ray measurements by the Thunderstorm Energetic Radiation Array (TERA) located at the ICLRT, Schaal et al. [2012] have indicated that energetic electrons, responsible for the production of these X-rays, exhibit a characteristic energy less than $3 \mathrm{MeV}$ and the maximum production rate of electrons is on the order of $10^{17}$ electrons/s. Additionally, on the basis of XCAM measurements of the structure of X-ray emissions from triggered lightning leaders, Schaal et al. [2014] have found that X-rays can originate from either diffuse or compact source regions and the electrical charge contained within the compact source region, typically with a radius between 2 and $3 \mathrm{~m}$, is on the order of $10^{-4} \mathrm{C}$.

Despite the above mentioned observational efforts, the exact production mechanism of X-rays during the stepping phase of lightning leaders is still not well understood. The mechanism of relativistic runaway electron avalanches (RREAs) in large-scale thunderstorm electric fields has been clearly ruled out as it cannot take place in the relatively small region in the vicinity of the leader tip, and it is not capable of explaining the observed energy spectra [Dwyer, 2004]. On the other hand, Celestin and Pasko [2011] have shown theoretically how the large flux of thermal runaway electrons generated by streamers during the negative corona flash stage of stepping lightning leaders could instead be responsible for these X-ray bursts during negative CGs and for terrestrial gamma ray flashes (TGFs) [Fishman et al., 1994] during intracloud lightning flashes (ICs). 


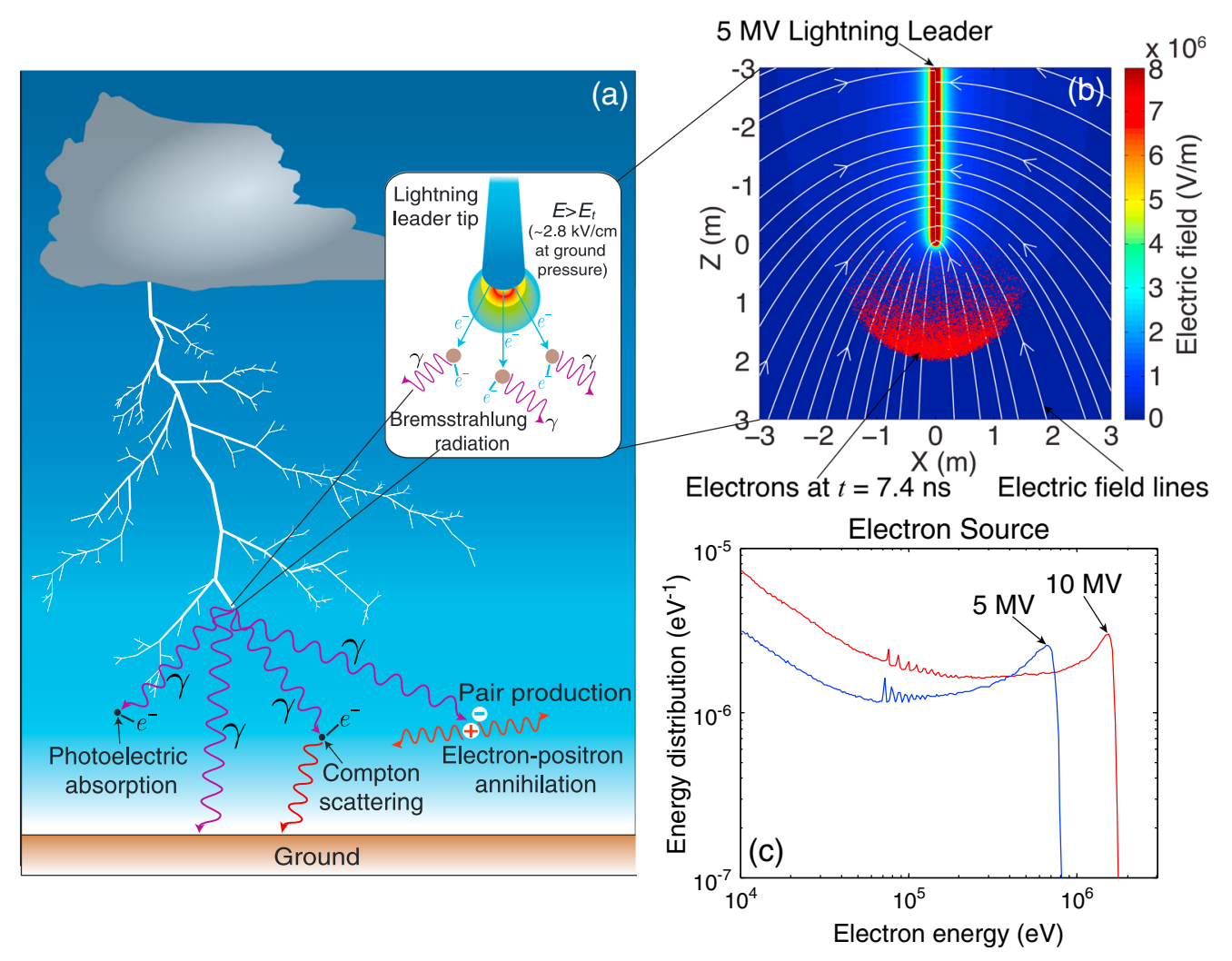

Figure 1. (a) Schematic of X-ray emissions produced during the stepping process of lightning leaders in a -CG flash, including acceleration of thermal runaway electrons in the electric field produced around lightning leader tip region, production of X-rays in bremsstrahlung emissions, and their further propagation in the Earth's atmosphere. (b) Simulation results for the inhomogeneous electric field produced by a $5 \mathrm{MV}$ lightning leader calculated using the method of moments and the positions of accelerating thermal runaway electrons at $t=7.4 \mathrm{~ns}$ derived from Monte Carlo simulation results. (c) Time-averaged electron energy distributions representing the acceleration of one thermal runaway electron in the electric fields produced around the tip region of stepping lightning leaders for two characteristic electrical potentials: $5 \mathrm{MV}$ and $10 \mathrm{MV}$. The curves are normalized so that the integration over the electron energy yields the amount of electrons generated per thermal runaway electron injected in the simulation. The results are obtained in air at ground level atmospheric density.

In this letter, based on the production mechanism of thermal runaway electrons by streamers during the negative corona flash stage of stepping lightning leaders [Celestin and Pasko, 2011], we simulate the further acceleration of these thermal runaway electrons in the electric field produced around lightning leader tips and predict the energy spectra of X-rays that can be observed. Using Monte Carlo simulations of photons propagating in the atmosphere from the source to the ground, we also investigate the properties of $\mathrm{X}$-rays that would be measured by a ground-based ideal detector during the stepping process of lightning leaders and estimate the effective radiation dose that humans may receive at ground in the nearby region.

\section{Model Formulation}

X-ray emissions produced by stepping -CG lightning leaders are modeled following a three-step procedure. First, we use the method of moments in order to calculate the electric field produced in the vicinity of lightning leader tips. Next, a Monte Carlo model is employed to simulate the dynamics of thermal runaway electrons in the lightning leader field obtained during the first step. Finally, from the differential cross section of bremsstrahlung radiation calculated using the Born approximation [Heitler, 1954, p. 245] without considering the screening effect and using a factorization of the angular and energy parts of the differential cross section [Lehtinen, 2000, pp. 45-49], we model the production of X-rays and their subsequent propagation in the Earth's atmosphere. The production mechanism and the relevant collisional processes are schematically depicted in Figure 1 a. 
The method of moments [Balanis, 1989, p. 670] is used to solve the integral equations of the electric potential and compute the electric field produced in the vicinity of a -CG lightning leader tip. For the sake of simplicity, we consider one lightning leader as a straight perfectly conducting wire. Stepping lightning leaders typically produce an electrical potential drop on the order of $10 \mathrm{MV}$ in the tip region [Bazelyan and Raizer, 2000, p. 106; Rakov and Uman, 2003, section 4.2, p. 111]. In the present work, we mainly study lightning leaders with two representative electrical potentials: $5 \mathrm{MV}$ and $10 \mathrm{MV}$. The electric potential of the lightning leader tip with respect to the ambient potential is approximately $V_{0}=E_{0} l / 2$ [Bazelyan and Raizer, 2000, p. 54], where $E_{0}$ is the ambient large-scale thunderstorm electric field, and $/$ is the length of the unbranched leader channel. In particular, the quantity $l$ is chosen as $1 \mathrm{~km}$ and the radius of the leader channel is $1 \mathrm{~cm}$ [Rakov and Uman, 2003, section 4.4.6, p. 134]. For a 5 MV leader, we assume an ambient large-scale thunderstorm electric field of $0.1 \mathrm{kV} / \mathrm{cm}$ [e.g., Marshall et al., 2001]. In the case of the $10 \mathrm{MV}$ leader, the ambient electric field is considered to be $0.2 \mathrm{kV} / \mathrm{cm}$ [e.g., Marshall et al., 2001].

In order to simulate the propagation and collisions of electrons in air under a given applied electric field, we use a Monte Carlo model, similar to that described in Celestin and Pasko [2011], which is three-dimensional (3-D) in the velocity space, 3-D in the configuration space, relativistic, and simulates electrons with energies from sub-eV to GeV. The initial location of the electrons is set at $15 \mathrm{~cm}$ from the leader tip in order to avoid the acceleration of electrons in unphysically high electric fields [see Celestin and Pasko, 2011]. The initial energy of the thermal runaway electrons is defined as $65 \mathrm{keV}$ because those are believed to be produced by streamer discharges developing in the vicinity of the leader tip during the negative corona flash process [Celestin and Pasko, 2011]. One notes that because of the strong acceleration of the thermal runaway electrons in the leader field, the exact value of the initial energy chosen is not important as long as the initial electrons are runaway in the leader field ( $>$ a few keVs). Bremsstrahlung radiation resulting from the deflection of energetic electrons by air molecules is then calculated using the time evolution of electron energy distributions. We note that X-rays produced by the initial production of thermal runaway electrons by streamers would have typical energies from a few tens of eVs to a few tens of keVs and would therefore be absorbed over distances shorter than $\sim 40 \mathrm{~m}$.

The Monte Carlo model developed to simulate photon transport through the atmosphere is similar to that described in Østgaard et al. [2008]. Three main collision types for photons with energies between $10 \mathrm{keV}$ and $100 \mathrm{MeV}$ are considered: Photoelectric absorption (main process for energies up to $30 \mathrm{keV}$ ), Compton scattering (main process from $\sim 30 \mathrm{keV}$ to $\sim 30 \mathrm{MeV}$ ), and electron-positron pair production (main process $>30 \mathrm{MeV}$ ). This model has been validated through calculations of TGF energy spectra based on RREA theory and a very good agreement has been obtained with previous published results [Dwyer and Smith, 2005; Xu et al., 2012].

Besides the stepping of lightning leaders, the attachment process is known to be associated with X-ray emissions [e.g., Howard et al., 2010]. Nevertheless, our present knowledge about the attachment processes is limited and, in this paper, we mainly focus on X-rays generated by the stepping process of lightning leaders. Specifically, it is considered that lightning leaders descend vertically in a series of discrete steps [Bazelyan and Raizer, 2000, p. 84] toward the ground and X-rays are not emitted between steps [Dwyer et al., 2005]. The step length of lightning leaders in -CGs typically measured in experiments is on the order of tens of meters [e.g., Rakov and Uman, 2003, Table 1.1]. It is chosen as $10 \mathrm{~m}$ in the present study. Note that this particular length has little effect on calculations of $X$-ray energy spectra received on the ground for radial distances greater than $\sim 30 \mathrm{~m}$. We also assume that X-rays produced after the formation of each new step follow the characteristic energy spectra of bremsstrahlung photons produced by the $5 \mathrm{MV}$ or $10 \mathrm{MV}$ lightning leaders as shown in Figure $1 \mathrm{c}$. As for the beaming of X-rays, it is assumed to be isotropic and within a downward solid angle of $2 \pi$, following the suggestion in [Saleh et al., 2009].

Schaal et al. [2012] observed the X-ray radiation of a -CG lightning event (MSE 10-01) using TERA. The stepped leader in this event had an average speed of $4.22 \times 10^{5} \mathrm{~m} / \mathrm{s}$ [e.g., Howard et al., 2010; Schaal et al., 2012]. They reported X-ray radiation in this event $891 \mu$ s prior to the time of return stroke, corresponding to the stepping of lightning leaders from approximately $376 \mathrm{~m}$ altitude to the ground [Schaal et al., 2012]. This range of altitudes (i.e., from $376 \mathrm{~m}$ to the ground) is adopted as a reference for the source locations of X-rays in our modeling. However, we note that the choice of source altitudes is not critical in this study, especially for analyzing the energy spectra of X-rays received at ground level for radial distances greater than $\sim 30 \mathrm{~m}$, because X-rays emitted from leader steps at relatively high altitudes are severely absorbed by the Earth's 

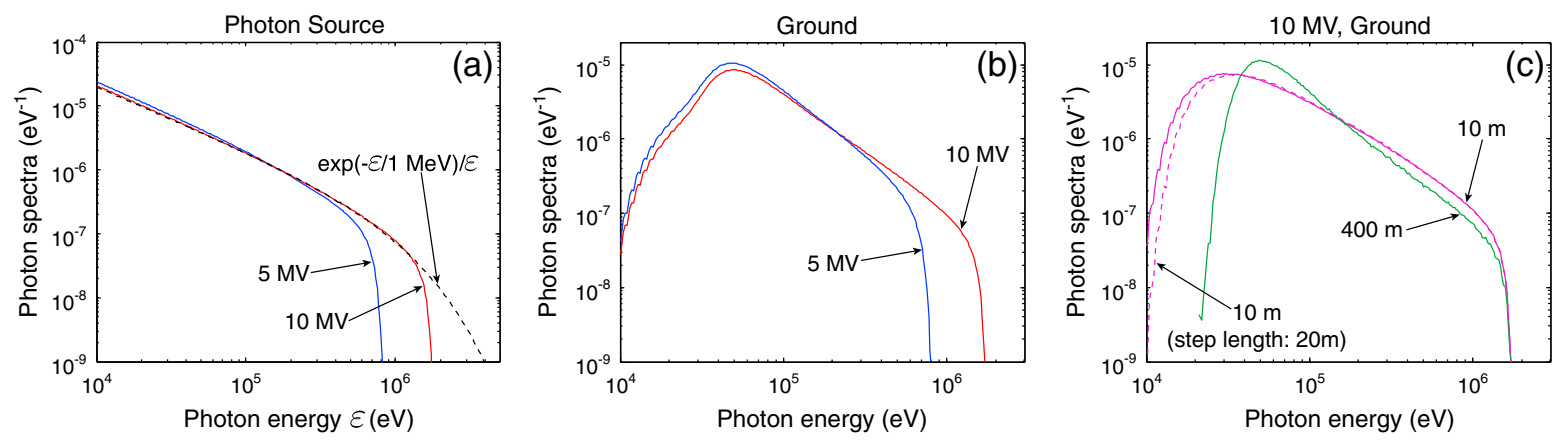

Figure 2. Photon energy spectra: (a) produced at the source by the bremsstrahlung radiation of energetic electrons accelerated in lightning leader fields for two electrical potentials: $5 \mathrm{MV}$ and $10 \mathrm{MV}$ (see Figure 1c); (b) collected at ground level within a radial distance of $500 \mathrm{~m}$ from the ground impact location of the lightning channel during the process of leader propagation from $376 \mathrm{~m}$ altitude to the ground. (c) Spectrum of photons collected at radial distances of $10 \mathrm{~m}$ and $400 \mathrm{~m}$ from the location of the $10 \mathrm{MV}$ lightning channel ground impact. The dashed line represents the energy spectra of photons collected at a radial distance of $10 \mathrm{~m}$ but for step lengths of the lightning leader assumed to be $20 \mathrm{~m}$.

atmosphere due to the high air density that they experience during their propagation towards the ground. It is also supported by the fact that X-ray radiation significantly intensifies as lightning leader approaches the ground [e.g., Schaal et al., 2014].

When evaluating the significance of radiation to living organisms, the effective radiation dose with units of Sievert (Sv), which is expressed in terms of joules of absorbed energy per kilogram, is usually used [Knoll, 2010; Dwyer et al., 2010]. In the present work, after obtaining the spatial energy distributions of X-rays at ground level, the effective radiation dose that can be received by the human body is calculated following the approach described in Dwyer et al. [2010, equation (2)]. In particular, the energy-dependent factors used for converting X-ray fluxes into radiation doses are obtained from Pelliccioni [2000].

\section{Results}

Figure $1 \mathrm{~b}$ shows modeling results for the inhomogeneous electric field produced in the vicinity of a $5 \mathrm{MV}$ lightning leader tip calculated using the method of moments and the positions of accelerating thermal runaway electrons at $t=7.4 \mathrm{~ns}$, which is chosen here for the sake of illustration, derived from the Monte Carlo simulation. As illustrated in this figure, the electric field produced by the $5 \mathrm{MV}$ lightning leader is highly inhomogeneous [e.g., Celestin et al., 2012] and the accelerating thermal runaway electrons are guided by the electric field lines. Figure 1c presents the time-averaged energy distribution functions obtained from the acceleration of thermal runaway electrons in the electric fields produced by stepping lightning leaders with two electric potentials: $5 \mathrm{MV}$ and $10 \mathrm{MV}$. The curves are normalized by first integrating over the time evolution of energy distribution and then dividing by the duration of the acceleration process and the number of electrons initially injected in the simulation. The integration of the distributions presented in Figure $1 \mathrm{c}$ over the electron energy yields the amount of electrons generated per thermal runaway electron injected in the simulation. We note that the maximum kinetic energy that thermal runaway electrons get in the electric field of the $10 \mathrm{MV}$ lightning leader is close to $2 \mathrm{MeV}$, resembling the most energetic X-rays typically observed by TERA [Saleh et al., 2009; Schaal et al., 2012]. In Figure 1c, the small peaks around $\sim 70 \mathrm{keV}$ are a consequence of the discreteness of the numerical integration of the spectra over time. Specifically, the peaks are produced by the initial group of thermal runaway electrons swiftly propagating in the energy space.

The energy spectra of bremsstrahlung photons at the source are further calculated and shown in Figure 2a using the time-averaged electron energy distributions presented in Figure 1c. One sees that the predicted energy spectra of X-rays corresponding to both $5 \mathrm{MV}$ and $10 \mathrm{MV}$ lightning leaders do not follow the theoretical energy distribution of bremsstrahlung photons produced by electrons with an exponential energy distribution and an $e$-folding kinetic energy. As expected, we also observe that the energy spectra of bremsstrahlung photons produced by a $10 \mathrm{MV}$ lightning leader are harder than those produced by a $5 \mathrm{MV}$ lightning leader.

Figure $2 \mathrm{~b}$ shows modeling results of the cumulative energy spectra of $\mathrm{X}$-rays measured by ideal detectors (without detector response) during leader propagation from $376 \mathrm{~m}$ altitude to the ground in the case when 
detectors are uniformly placed per unit area within a radial distance of $500 \mathrm{~m}$ from the ground impact location of the lightning channel. A distance of $500 \mathrm{~m}$ is chosen to represent the detecting range covered by TERA [e.g., Saleh et al., 2009]. Figure 2c presents the X-ray energy spectra in the $10 \mathrm{MV}$ lightning leader case as measured by an ideal detector at radial distances of $10 \mathrm{~m}$ and $400 \mathrm{~m}$ from the lightning channel ground impact. To obtain these results, we have accumulated the partial spectra of those shown in Figure $2 \mathrm{~b}$ over concentric rings defined on the ground at radial distances between 8 and $12 \mathrm{~m}$, and 398 and $402 \mathrm{~m}$ for the distances of $10 \mathrm{~m}$ and $400 \mathrm{~m}$, respectively. We have verified that these rings are sufficiently thin so that the spectra vary only negligibly from the inner radius to the outer radius of each ring.

The dashed line in Figure $2 \mathrm{c}$ also shows the energy spectra of photons collected at the radial distance of $10 \mathrm{~m}$ but for the case that the step length of the lightning leader is $20 \mathrm{~m}$, representing the uncertainties caused by the choice of leader step length (specifically, quantitative deviations observed in the energy range below $50 \mathrm{keV}$ ). For detectors located very close to the ground impact location of the lightning channel (i.e., within a few tens of meters), the recorded X-rays mostly originate from the last leader step, before the attachment process. This is mainly due to the fact that the decreased distance between the $\mathrm{X}$-ray source and detectors enables a large population of photons with energies of a few tens of keVs to reach detectors without being absorbed in the air. For instance, the mean free path for $\mathrm{X}$-rays with an energy of $20 \mathrm{keV}$ is $\sim 13 \mathrm{~m}$ at ground level air density, and therefore, one should observe an enhancement in the spectrum in this range of energy for detectors located at a radial distance of $10 \mathrm{~m}$ if the step length decreases from $20 \mathrm{~m}$ (corresponding to the last step terminating at $16 \mathrm{~m}$ altitude) to $10 \mathrm{~m}$ (with the last step at $6 \mathrm{~m}$ altitude) as evident in Figure $2 c$. However, we note that the choice of step length hardly changes the energy spectra of X-rays collected, for example, at a radial distance of $400 \mathrm{~m}$. More interestingly, the X-ray energy spectra show a significant radial dependence: as the distances from lightning channel decrease, the energy spectrum extends to the range below $\sim 50 \mathrm{keV}$ and becomes harder in the range above $\sim 50 \mathrm{keV}$.

\section{Summary and Discussion}

In this work, we have simulated the dynamics of thermal runaway electrons accelerating in the inhomogeneous electric field produced around lightning leader tips [Celestin and Pasko, 2011] to study the production of bremsstrahlung X-ray bursts from -CG lightning discharges. Using Monte Carlo simulations of the transport of these X-rays down to the ground, we predict the characteristic time-averaged electron energy distributions produced by $5 \mathrm{MV}$ and $10 \mathrm{MV}$ lightning leaders and the corresponding raw energy spectra of $\mathrm{X}$-rays that can be observed at ground level caused by the stepping process of lightning leaders in -CGs.

The energy distributions of electrons and resultant $\mathrm{X}$-rays obtained from the acceleration of thermal runaway electrons in lightning leader fields are directly related to physical properties of the stepping lightning leaders [e.g., Xu et al., 2012; Celestin et al., 2012]. This point is illustrated by the comparison of source X-ray energy spectra presented in Figure $2 a$. We observe that the maximum energy that electrons acquire from the $10 \mathrm{MV}$ lightning leader potential, which is defined by the electric potential drop in the leader tip region, is approximately twice that of the $5 \mathrm{MV}$ lightning leader. Moreover, the hardness of $\mathrm{X}$-ray energy spectra is also different between the two leader potentials. In contrast to lightning leaders in -CGs, long unbranched lightning leaders in ICs generally possess larger electric potentials and have been demonstrated to be able to generate gamma rays with energies up to a few tens of $\mathrm{MeV}$ and explain satellite measurements of TGF spectra [Xu et al., 2012; Celestin et al., 2012]. Due to the correlation between the photons and the causative potential drop produced by lightning leaders, measurements of $X$-ray radiation can bring a significant insight on the electrical properties of lightning leaders.

Modeling results of $\mathrm{X}$-ray energy spectra received at ground by ideal detectors during the stepping process of lightning leaders are comparable to TERA measurements in terms of general shape and spectral hardness [Schaal et al., 2012]. However, detailed comparison with TERA measurements should take detector response into consideration. As the radial distance from the lightning channel increases, photons received at ground, on average, experience more scatterings by air molecules. Given that photons are completely absorbed in photoelectric absorption and lose considerable energy in Compton scattering, energy spectra of photons received at ground should demonstrate significant radial dependence as evident in Figure $2 \mathrm{c}$. The sharp decrease in the energy region below $\sim 50 \mathrm{keV}$ stems from the fact that the increased amount of photoelectric absorption hampers low-energy photons from reaching further radial distances. As for the change in the 
energy spectrum above $\sim 50 \mathrm{keV}$, it primarily reflects the fact that photons undergo more Compton scatterings to reach further radial distances, which significantly softens the energy spectra. Furthermore, we note that the $511 \mathrm{keV}$ positron annihilation line is not characteristics for the phenomenon discussed in this paper. This is mainly because of the fact that photons typically produced by stepping lightning leaders in -CGs have energies up to a few $\mathrm{MeV}$ and electron-positron pair production is not the dominant collisional process in this energy range.

Based on XCAM measurements, Schaal et al. [2014] have suggested that the compact source region in front of lightning leaders, responsible for production of X-rays, has a radius between 2 and $3 \mathrm{~m}$ and the electric charge contained in this region is on the order of $10^{-4} \mathrm{C}$. From the quadratic relation between the electric charge contained in the streamer zone and the electrical potential of the associated lightning leader [Celestin and Pasko, 2011], we estimate from first principles that the charge in the streamer zone of a $5 \mathrm{MV}$ lightning leader is approximately $2.8 \times 10^{-4} \mathrm{C}$. As for the $10 \mathrm{MV}$ lightning leader, the electric charge in the streamer zone is $1.1 \times 10^{-3} \mathrm{C}$. We also estimate the radius of the streamer zone in front of the $5 \mathrm{MV}$ and $10 \mathrm{MV}$ lightning leaders and the values are, respectively, $2 \mathrm{~m}$ and $4 \mathrm{~m}$. Both the electric charge contained in the X-ray source region and the size of the streamer zone are consistent with results in Schaal et al. [2014].

Using the singly differential cross section of bremsstrahlung emissions and estimating the number of initial thermal runaway electrons injected by steamers in the leader field from the method derived in Celestin and Pasko [2011], we find that a $10 \mathrm{MV}$ lightning leader should produce less than $10^{15}$ photons (upper limit) with energies above $10 \mathrm{keV}$. From this number, we evaluate that the maximum radiation exposure at ground level, corresponding to the ground impact location of the lightning channel, as produced during the stepping process of a $10 \mathrm{MV}$ lightning leader during its propagation down to the ground is $\sim 0.4 \mathrm{mSv}$. Given that $\sim 12 \mathrm{mSv}$ is the effective radiation dose for a full-body computerized tomography scan [e.g., Brenner and Elliston, 2004], this amount of radiation is considered to be insignificant to humans.

\section{Acknowledgments}

This research was supported by the NSF grants AGS-1106779 and AGS-0741589 to Pennsylvania State University. Sebastien Celestin's research was supported by the French space agency (CNES). All data used in this paper are directly available after a request is made to authors W.X., S.C., or V.P.P.

W.K. Peterson thanks two anonymous reviewers for their assistance in evaluating this paper.

\section{References}

Balanis, C. A. (1989), Advanced Engineering Electromagnetics, John Wiley, New York.

Bazelyan, E. M., and Y. P. Raizer (2000), Lightning Physics and Lightning Protection, Instit. of Phys., Bristol, Pa.

Brenner, D. J., and C. D. Elliston (2004), Estimated radiation risks potentially associated with full-body CT screening, Radiology, 232, 735-738, doi:10.1148/radiol.2323031095.

Celestin, S., and V. P. Pasko (2011), Energy and fluxes of thermal runaway electrons produced by exponential growth of streamers during the stepping of lightning leaders and in transient luminous events, J. Geophys. Res., 116, A03315, doi:10.1029/2010JA016260.

Celestin, S., W. Xu, and V. P. Pasko (2012), Terrestrial gamma ray flashes with energies up to $100 \mathrm{MeV}$ produced by nonequilibrium acceleration of electrons in lightning, J. Geophys. Res., 117, A05315, doi:10.1029/2012JA017535.

Dwyer, J. R. (2004), Implications of X-ray emission from lightning, Geophys. Res. Lett., 31, L12102, doi:10.1029/2004GL019795

Dwyer, J. R., and D. M. Smith (2005), A comparison between Monte Carlo simulations of runaway breakdown and terrestrial gamma-ray flash observations, Geophys. Res. Lett., 32, L22804, doi:10.1029/2005GL023848.

Dwyer, J. R., et al. (2003), Energetic radiation during rocket-triggered lightning, Science, 299, 694-697, doi:10.1126/science.1078940.

Dwyer, J. R., et al. (2004), Measurements of x-ray emission from rocket-triggered lightning, Geophys. Res. Lett., 31, L05118, doi:10.1029/2003GL018770.

Dwyer, J. R., et al. (2005), X-ray bursts associated with leader steps in cloud-to-ground lightning, Geophys. Res. Lett., 32, L01803, doi:10.1029/2004GL021782.

Dwyer, J. R., D. M. Smith, M. A. Uman, Z. Saleh, B. Grefenstette, B. Hazelton, and H. K. Rassoul (2010), Estimation of the fluence of high-energy electron bursts produced by thunderclouds and the resulting radiation doses received in aircraft, J. Geophys. Res., 115, D09206, doi:10.1029/2009JD012039.

Dwyer, J. R., M. Schaal, H. K. Rassoul, M. A. Uman, D. M. Jordan, and D. Hill (2011), High-speed X-ray images of triggered lightning dart leaders, J. Geophys. Res., 116, D20208, doi:10.1029/2011JD015973.

Fishman, G. J., et al. (1994), Discovery of intense gamma-ray flashes of atmospheric origin, Science, 264(5163), 1313-1316.

Heitler, W. (1954), The Quantum Theory of Radiation, 3rd ed., Clarendon, Oxford, U. K.

Howard, J., M. A. Uman, J. R. Dwyer, D. Hill, C. Biagi, Z. Saleh, J. Jerauld, and H. K. Rassoul (2008), Co-location of lightning leader x-ray and electric field change sources, Geophys. Res. Lett., 35, L13817, doi:10.1029/2008GL034134.

Howard, J., M. A. Uman, C. Biagi, D. Hill, J. Jerauld, V. A. Rakov, J. Dwyer, Z. Saleh, and H. Rassoul (2010), RF and X-ray source locations during the lightning attachment process, J. Geophys. Res., 115, D06204, doi:10.1029/2009JD012055.

Knoll, G. F. (2010), Radiation Detection and Measurement, 4th ed., John Wiley, Hoboken, N. J.

Lehtinen, N. G. (2000), Relativistic runaway electrons above thunderstorms, PhD thesis, Stanford Univ., Stanford, Calif.

Marshall, T. C., M. Stolzenburg, W. D. Rust, E. R. Williams, and R. Boldi (2001), Positive charge in the stratiform cloud of a mesoscale convective system, J. Geophys. Res., 106(D1), 1157-1164, doi:10.1029/2000JD900625.

Moore, C. B., K. B. Eack, G. D. Aulich, and W. Rison (2001), Energetic radiation associated with lightning stepped-leaders, Geophys. Res. Lett., 28(11), 2141-2144, doi:10.1029/2001GL013140.

Østgaard, N., T. Gjesteland, J. Stadsnes, P. H. Connell, and B. Carlson (2008), Production altitude and time delays of the terrestrial gamma flashes: Revisiting the Burst and Transient Source Experiment spectra, J. Geophys. Res., 113, A02307, doi:10.1029/2007JA012618.

Pelliccioni, M. (2000), Overview of fluence-to-effective dose and fluence-to-ambient dose equivalent conversion coefficients for high energy radiation calculated using the FLUKA code, Radiat. Prot. Dosim., 88, 279-297.

Rakov, V. A., and M. A. Uman (2003), Lightning: Physics and Effects, Cambridge Univ. Press, Cambridge, U. K. 
Saleh, Z., J. Dwyer, J. Howard, M. Uman, M. Bakhtiari, D. Concha, M. Stapleton, D. Hill, C. Biagi, and H. Rassoul (2009), Properties of the X-ray emission from rocket-triggered lightning as measured by the Thunderstorm Energetic Radiation Array (TERA), J. Geophys. Res., 114, D17210, doi:10.1029/2008JD011618.

Schaal, M. M., J. R. Dwyer, Z. H. Saleh, H. K. Rassoul, J. D. Hill, D. M. Jordan, and M. A. Uman (2012), Spatial and energy distributions of X-ray emissions from leaders in natural and rocket triggered lightning, J. Geophys. Res., 117, D15201, doi:10.1029/2012JD017897.

Schaal, M. M., et al. (2014), The structure of X-ray emissions from triggered lightning leaders measured by a pinhole-type X-ray camera, J. Geophys. Res. Atmos., 119, 982-1002, doi:10.1002/2013JD020266.

Xu, W., S. Celestin, and V. P. Pasko (2012), Source altitudes of terrestrial gamma-ray flashes produced by lightning leaders, Geophys. Res. Lett., 39, L08801, doi:10.1029/2012GL051351. 